\title{
Update on musculoskeletal magnetic resonance imaging, dedicated to the first honourable member of the Polish Medical Radiological Society Madame Maria Sklodowska-Curie
}

\author{
Mihra S. Taljanovic \\ Emeritus Professor, Departments of Medical Imaging and Orthopaedic Surgery, University of Arizona, Banner University Medical Centre, Tucson, \\ Arizona, USA
}

Since its development in the 1970s and 1980s, magnetic resonance imaging (MRI) has become a widely used imaging modality in the evaluation of various musculoskeletal (MSK) traumatic and pathological conditions. Subsequent rapid improvement of the standard and development of functional MRI techniques and sequences on high-resolution MRI scanners has led to superb contrast resolution imaging of various MSK structures including bones, joints, muscles, tendons, ligaments, and nerves. This series of nine review MRI articles provides an update on MSK MRI in routine clinical practice with an emphasis on normal anatomy and various traumatic and pathologic conditions.

The authors are honoured to dedicate this MSK MRI series to the heroic female scientist and proud daughter of Poland Madame Maria Sklodowska-Curie, who was a double recipient of the Nobel Prize. She received the award first in 1903 for physics with her husband Pierre Curie and Henri Becquerel, for their work in radioactivity. She received it again in 1911 for chemistry, for the discovery of two radioactive elements, Polonium and Radium. As the author of this Editorial I would like share with the readership - a personal connection with this female scientist. It was a long time ago when, as a 10-year-old girl in former Yugoslavia, I had an opportunity to read a biography of Maria Sklodowska-Curie written by her daughter, Ewa Curie. Since then, I was and remain to be amazed by this impressive scientist - the first woman to win the Nobel Prize and the first person to win it twice, while remaining the only one to receive it in two separate sciences. She became my idol, a woman I admire, and the main inspiration for choosing my career in radiology. This impressive female scientist will live forever through her incredible and invaluable contribution to science. Naming one of the radioactive elements she discovered Polonium confirmed how proud she was of her Polish heritage, and the fact that she became the first honourable member of the Polish Medical Radiological Society confirms how much Poland is proud of her. As a Slavic-born woman radiologist, I must admit that I am proud of her success, as well. I hope the admirable Polish scientist Madame Maria Sklodowska-Curie will continue to inspire future generations of radiologists and scientists as she inspired me.

Now, I would like to introduce to the readership a series of nine articles entitled "Update on musculoskeletal magnetic resonance imaging", which includes the evaluation of major upper and lower extremity joints, spine and bone, and soft tissue tumors.

Two highlights of this series are the articles on musculoskeletal tumor imaging by Doctors Ahlawat and Fayad from Johns Hopkins University, Baltimore, Maryland. The title of the first article is "Revisiting the WHO classification system of soft tissue tumors: emphasis on advanced magnetic resonance imaging sequences. Part 1", and the second is "Revisiting the WHO classification system of bone tumors: emphasis on advanced magnetic resonance imaging sequences. Part 2". The authors discuss the most recent updates to the WHO classification of the soft tissue and bone tumors, which are relevant to radiologists in routine clinical practice, with MRI correlation. They highlight the utility of advanced MRI sequences such as diffusion-weighted imaging, dynamic contrast enhanced

Correspondence address:

Mihra S. Taljanovic, MD, PhD, FACR, Emeritus Professor, Department of Medical Imaging, The University of Arizona, College of Medicine, 1501 N. Campbell Ave.,

P.O. Box 245067, Tucson, AZ 85724, phone: 520 626-9077, fax: 520 626-1945, e-mail: mihrat@radiology.arizona.edu 
sequences, and magnetic resonance spectroscopy, to provide insight into the biological behaviour of various tumors.

In their manuscript "Magnetic resonance imaging of the shoulder", Doctors Ashir, Lombardi, Jerban, Ma, and Chang for the University of California, San Diego review imaging techniques, normal anatomy, and the most common imaging findings of disorders of tendons, labrum, and ligaments of the shoulder. They discuss common systemic diseases that affect the shoulder and the disorders of the acromioclavicular joint and bursae. New advances and research in MRI have provided additional potential uses for evaluating shoulder derangements. In particular, the newer MRI techniques, such as zero echo time (ZTE) or inversion-recovery (IR) UTE imaging, can be used to generate high-contrast bone images. ZTE provides an isotropic dataset, which facilitates the creation of 3D-volume rendered images, but IR-UTE provides better suppression of non-bone tissues.

The article "Magnetic resonance imaging of the elbow" is a joint effort of authors from three American universities: University of California, San Diego; Drexel University College of Medicine, Philadelphia, Pennsylvania; and the University of Arizona, Tucson, Arizona. Doctors Lombardi, Ashir, Gorbachova, Taljanovic, and Chang describe the normal MRI anatomy, biomechanics, commonly used imaging techniques, and the common MRI findings related to tendinopathy, ligamentous and osteochondral injuries, and instability of the elbow joint.

In their article "Magnetic resonance imaging of the wrist and hand", Doctors Vassa, Garg, and Omar from Northwestern University Feinberg School of Medicine, Chicago, Illinois describe the normal anatomy of the wrist and hand complex, imaging techniques, and MRI findings of various traumatic and pathologic conditions of the wrist and hand, including occult fractures, osteonecrosis, ligamentous and tendon injuries, and entrapment neuropathies.

Normal MRI anatomy, imaging protocols, and common painful conditions of various etiologies of the hip joint that affect primarily the adult population are addressed in the article "Magnetic resonance imaging of the hip: anatomy and pathology" by Doctors May San Mak and Teh from Nuffield Orthopaedic Centre, Oxford University Hospitals NHS Trust, United Kingdom. The authors divided hip pathologies by their location into osseous, intra-articular, and extra-articular lesions. Among other common traumatic and pathologic conditions, they discuss various types of femoroacetabular impingement, including cam, pincer and mixed morphology, femoral torsion abnormalities, iliopsoas, and subspine impingement.

The article "Magnetic resonance imaging of the knee" is a joint effort from authors from three American universities: Loma Linda University Medical Centre, Murrieta, California; University of New Mexico Health Sciences, Albuquerque, New Mexico; and Northwestern University
Feinberg School of Medicine, Chicago, Illinois. This article by Doctors Chien, Weaver, Kinne, and Omar reviews the current clinical practice of the evaluation and interpretation of the meniscus, ligamentous, and cartilaginous structures, as well as other non-tumor pathologies that are commonly encountered on MRI of the knee.

In her article "Magnetic resonance imaging of the ankle and foot" Dr. Gorbachova from Drexel University College of Medicine, Philadelphia, Pennsylvania discusses the MRI imaging protocols, normal anatomy, and various traumatic and pathologic conditions of the ankle and foot commonly encountered in clinical practice. This includes osseous and osteochondral injuries, ligamentous injuries, common traumatic and degenerative tendon pathology, abnormalities of the Chopart and Lisfranc joints complexes, pathological conditions affecting capsuloligamentous structures of the great toe and lesser toes, as well as pedal infection, with a focus on diabetic osteomyelitis and neuropathic osteoarthropathy.

"Magnetic resonance imaging of the spine" by Doctors Winegar, Kay, and Taljanovic from the University of Arizona reviews normal MRI anatomy of the spine and various imaging findings associated with degenerative disc disease with an emphasis on spine location reporting, facet and uncovertebral joint arthrosis, synovial cyst, spinal canal, subarticular recess and neural foraminal narrowing, spondylolysis and spondylolisthesis, Baastrup syndrome and interspinal posterior epidural cyst, and pyogenic and tuberculous spine infection. The authors discuss the imaging findings of vertebral insufficiency and pathologic fractures with an emphasis on how to differentiate between the vertebral insufficiency fractures and malignant pathologic fractures.

As an author of this Editorial, I thank all the authors for spending a substantial amount of time writing these valuable review articles and sharing their knowledge and expertise with the readers of the Polish Journal of Radiology. I would also like to thank the Editor-in-Chief of the Polish Journal of Radiology, Professor Marek Sasiadek, for the invitation and for providing guidance for this MSK MRI series. 